\title{
Letting a Good Crisis Go to Waste
}

\author{
Andrew D. Auerbach, MD, MPH ${ }^{1,2}$, Raman Khanna, $\mathrm{MD}^{1,2}$, and Julia Adler-Milstein, \\ $P h D^{1,3}$
}

'San Francisco Division of Hospital Medicine, University of California, San Francisco, CA, USA; ${ }^{2}$ San Francisco Center for Digital Health Innovation, University of California, San Francisco, USA; ${ }^{3}$ San Francisco Center for Clinical Informatics \& Improvement Research, University of California, San Francisco, USA.

The widespread implementation of electronic health records (EHRs) was predicated on hopes that they would rapidly improve care, but initial experiences have been disappointing and thought to be a key part of physician dissatisfaction and burnout. The crisis created by EHR implementation is only in part due to EHRs themselves, and might also be viewed as a crisis that has served to surface longstanding problems in healthcare-ones that if grappled with, will lead to more rapidly effective digital transformation.

J Gen Intern Med 35(4):1289-91

DOI: $10.1007 / \mathrm{s} 11606-019-05552-\mathrm{Z}$

(c) Society of General Internal Medicine 2019

I $\mathrm{n}$ the past 7 years, the majority of health systems in the USA have transitioned to electronic health record (EHR) systems. The profound effects of EHR implementation on the work of healthcare professionals have been a core part of a crisis described repeatedly in recent years. ${ }^{1}$

Troubles ascribed to EHRs are valid. However, current discussions often forget or gloss over the distinction between problems EHRs create and preexisting ones that EHRs have simply made more visible. If we routinely lump these two forms of problems together rather than reframing them both as core challenges to healthcare, we are letting an important crisis - a crisis of physician and provider burnout, of loss of connection to patients and peers due to electronic tools, and of widespread dissatisfaction with EHRs - go to waste. We provide examples of complaints that represent latter aspects of the crisis, and point out how they provide opportunities for reframing and addressing fundamental features of healthcare that have been surfaced by the arrival of EHRs (Table 1).

Received June 4, 2019

Revised September 22, 2019

Accepted October 31, 2019

Published online November 19, 2019

\section{OUR EHR DOESN'T IMPROVE CARE AS RAPIDLY AS WE WOULD LIKE}

Care improvement efforts have always been constrained both by the tools available to carry out the change. In the digital era, EHRs have expanded access to key improvement resources (such as data), while also raising hopes that - by centralizing improvement opportunities in a ubiquitous system — quality improvement and safety enhancement would be greatly facilitated.

Sadly, the pace of improvement remains slow and EHRs' ability to improve care has proven more limited than hoped. However, limitations in quality improvement tools have always limited the scope of our goals for the interventions themselves. What may be different in the EHR era is the expectations from many users that simple changes in EHRs will lead to instant changes in care quality. Providers and improvement teams need to recommit to core methods of quality improvement and harm reduction and consider how EHR solutions are best developed as components of larger initiatives, rather than treating EHR programs as a simple flip of the switch (or by pushing all "improvement" work to the front line providers in the form of extensive documentation requests or hard-stops). Stated alternately, while EHRs are a key part of quality improvement, EHRs are usually not the first or sole part of an effective improvement strategy.

\section{OUR EHR IS TOO COMPLEX TO BE USEFUL}

EHRs suffer by comparison to websites and apps we relish using in our everyday lives. A disconnect between consumerfacing digital apps and websites drives dissatisfaction with EHRs, and prompts requests for customization and add-ons that meet individual's needs, but which only add further complexity to the EHR.

However, EHR complexity is a problem of healthcare's own making. Vendors of EHRs have built software in response to requests to accommodate an incredibly diverse set of wishes from customers at a large number of healthcare settings. As a result, the complexity of the software provided by vendors represents healthcare's larger demands for flexibility.

Even within hospitals and health systems, EHRs are configured in ways that meet the needs of many possible personnel 
Table 1 Reactions and Reframed Responses to Electronic Health Record Systems

\begin{tabular}{|c|c|}
\hline Reaction to EHR use & Reframed current state \\
\hline $\begin{array}{l}\text { Our EHR doesn't improve care as rapidly as we would } \\
\text { like. } \\
\text { Our EHR is too complex to be useful. }\end{array}$ & $\begin{array}{l}\text { - Resources and staff dedicated to EHR improvements are limited and may need to be } \\
\text { prioritized. } \\
\text { - EHRs are attempting to represent complex processes that vary both within and across } \\
\text { providers and institutions. }\end{array}$ \\
\hline $\begin{array}{l}\text { The EHR should be able to accommodate the wide range } \\
\text { of clinical decisions I need to make. }\end{array}$ & - Reducing variation in clinical care also represents an opportunity to improve the EHR. \\
\hline $\begin{array}{l}\text { The EHR exists primarily to support checkbox tasks and } \\
\text { billing. }\end{array}$ & $\begin{array}{l}\text { - Billing and other clerical tasks are not likely to go away, so approaches to reducing } \\
\text { burden on providers may require reexamination of how and why such tasks are carried } \\
\text { out or consider supporting them outside the EHR. }\end{array}$ \\
\hline Documentation is all I do. & $\begin{array}{l}\text { - In order to reduce documentation burden, it may be most important to first consider } \\
\text { what information needs to be documented in each note or should be discoverable in the } \\
\text { EHR. }\end{array}$ \\
\hline
\end{tabular}

and processes. ${ }^{2}$ In this way, seemingly unnecessary complexity in the EHR is the response to how many ways we do our work; it also places huge strains on informatics teams to create more and more customized tools. While customization is likely a good approach in some cases, if we want the elegance of commercial websites (none of which deals with processes nearly as complex as healthcare), we must first standardize and simplify our electronic and non-electronic work. Only then we can simplify our digital workspaces accordingly.

\section{THE EHR SHOULD BE ABLE TO ACCOMMODATE THE WIDE RANGE OF CLINICAL DECISIONS I NEED TO MAKE}

Deciding on the best treatment or diagnostic test for a patient requires consideration of many options. EHRs can efficiently warehouse an infinite number of diagnostic and therapeutic choices, but without curation, the EHR warehouse quickly becomes cluttered. For example, order sets can be tailored to primarily meet the goal of "which medication do I frequently order?" or the goal of "how can I select medications that are safe and high-value?" The balance between these two viewpoints represents another key tension in the EHR era: presenting many or all potential clinical choices, and guiding caregivers towards the best care possible.

These tensions are not new: Challenges in defining best clinical practices, gaining consensus, and then moving these realizations into practice have been longstanding. What has happened in the EHR era is that adaptations to dealing with a perceived lack of flexibility keep us from the standardized solution for which EHRs are extraordinarily well suited, while also adding to the complexity and lack of usefulness we described earlier.

\section{THE EHR EXISTS PRIMARILY TO SUPPORT CHECKBOX TASKS AND BILLING}

Implementation of EHRs has brought to light checkbox activities that were carried out using implicit standards (e.g., rubber stamps to substitute for physician signatures), workarounds (e.g., verbal orders), or the need for supervising physicians to cosign or attest to trainees' documentation. However, making tasks explicit and auditable was a desired outcome from the transition to EHRs and in some cases has made care safer by reinforcing best practices and providing a platform where roles and tasks are clear to all involved.

Issues around billing requirements and associated documentation and compliance tasks are real, but are not solely of EHRs' making. Much as EHR vendors have had to adapt to multiple different workflows in order to produce a product viable in the marketplace (our first point), they have also had to simultaneously deal with rapidly evolving and idiosyncratic rules for billing and their customers' adaptations to and interpretations of those rules. As a result, improved adherence to billing requirements is the worst-case scenario of EHR clerical tasks. However, billing is a major part of EHR work burden not because EHR vendors are unsophisticated - but because their product needs to ensure that hospitals and physicians would continue to be paid.

\section{DOCUMENTATION IS ALL I DO}

Separate from the need to document for billing purposes is our need to document in order to create a durable record of a patient's treatments and relay this information to ourselves or to the next provider. When referral letters, progress or consultation notes, and discharge summaries were only available on paper, they were the sole means for this form of communication, easier to create (via dictation and transcription), certainly more tailored to providers' needs, and in some ways more easily searched through in physical form because the volume of information gathered in paper form was lower and because health systems had developed workarounds such as "shadow charts" representing subsets of patient information relevant to groups of providers but unfindable to others.

EHRs have had multiple negative effects on the work of creating notes - many of which relate to billing and checkbox clerical tasks. The ability to automatically pre-populate notes with complex clinical data, as well as cut-and-paste, is an adaptation to documentation burden, but makes notes both less useful and more complicated. 
Our EHR notes digitally reproduce our old ideas of "good" clinical communication between providers, rather than an act of curating patient-centered care. Solutions such as scribes or artificial intelligence-enabled transcription and searches will save time by finding clinical data more quickly, creating distilled notes, or helping ensure that key aspects of clinical data collection or decision-making are documented. But we need to grapple with simple things - like divergence in providers' views of which information needs to be included in a discharge summary ${ }^{3-5}$ - so that new tools can be deployed most effectively. More fundamentally, we should reconsider how information should be gathered, transcribed, or entered into the EHR. Which should be relayed in documents at all? Which are best found in standard places in electronic systems? And who should receive which information via a "note," when the information is readily available in the EHR?

\section{CONCLUSIONS}

We are not writing this piece as an apology for EHR vendors, or to suggest that effects on morale and burnout are not valid - far from it. Both sets of issues are real, well-documented, and critical to address. However, we need to recognize how approaches to healthcare improvement and the culture of medicine both add to and are amplified by EHRs' limitations, leading to the overarching theme to our reframing: Healthcare needs to pay attention to this relationship, or the crisis we are currently experiencing will be prolonged. We see several ways forward.

The key first step is recognizing our role in making the EHR what it has become. At a fundamental level, EHRs are a representation of local standards and practices even if delivered by a software vendor. In this reframing, many aspects of the "EHR-as-enemy" narrative can be reframed as signaling unfinished business for our hospitals and clinics. Healthcare must pay greater attention to the leadership, management, and policy work needed to update practices and standards so that they can be represented more effectively in the digital era, rather than using digital tools to represent paper-based standards, recreate past controversies, or simply act as a cluttered warehouse of various potential tools and suggestions.

Health systems must grapple with how the EHR can be a platform upon which improvement and standardization takes place, rather than the sole method for achieving change. We remain optimistic that improvement will accelerate rapidly once this holistic view is embraced. However, whether the goal is reducing documentation burden or improving a clinical outcome, process redesign and measuring change both inside and outside the EHR remain critical components of successful programs. For example, work tasks surfaced in the EHR era might be better represented as part of a scope of practice, rather than requiring an order or "approval"; the EHR can then be used for decision support to facilitate the process and help everyone practice at the "top of their license."
While EHR technical limitations can be a core issue, they are potentially a handy set of excuses for what may be better described as an EHR governance failure. In our view, future state EHR governance needs to first standardize practices and workflows and focus on the EHR as a "platform" for spread of best practices. Becoming an improvement platform for agreed-upon work and clinical practices leads to a holistic improvement strategy that emphasizes spread, usability, ongoing training, and clinical impact rather than customization and complexity acting as band-aids over longstanding problems.

Governing will mean understanding needs of patients and providers, intense focus on training and support, and close collaboration with the EHR team not as technicians but as collaborators and consultants. Importantly, these discussions will - by their very nature - better integrate the "IT" world with the clinical care delivery world, and we would encourage this thinking as a core tenet going forward. EHR vendors need to be responsive to requests for modifications that result from governance, and likely have a key role to play in supporting governance approaches as a larger part of their ongoing relationships with their customers; this role will need to change towards being focused on helping sites pick platforms and solutions that work across problems, rather than supporting customizations as a primary customer service goal.

The future is bright and opportunities of the digital era nearly unbounded. However, our current crisis will be wasted and the bright future delayed, if healthcare misses the opportunity to link digital reinvention to clinical process reinvention.

Corresponding Author: Andrew D. Auerbach, MD, MPH; San Francisco Division of Hospital Medicine, University of California, San Francisco, CA, USA (e-mail: ada@medicine.ucsf.edu).

\section{Compliance with Ethical Standards:}

Conflict of Interest: Raman Khanna helped develop CareWeb, an electronic communication product which has been licensed by Voalte, Inc. The authors declare no other conflicts of interest in relationship to this article.

\section{REFERENCES}

1. Downing N, Bates DW, Longhurst CA. Physician burnout in the electronic health record era: Are we ignoring the real cause? Ann Intern Med 2018.

2. Vaughn VM, Linder JA. Thoughtless design of the electronic health record drives overuse, but purposeful design can nudge improved patient care. BMJ Qual Saf 2018.

3. Sheu L, Fung K, Mourad M, Ranji S, Wu E. We need to talk: Primary care provider communication at discharge in the era of a shared electronic medical record. J Hosp Med 2015;10:307-10.

4. Sorita A, Robelia PM, Kattel SB, et al. The Ideal Hospital Discharge Summary: A Survey of U.S. Physicians. J Patient Saf 2017.

5. van Walraven C, Rokosh E. What is necessary for high-quality discharge summaries? Am J Med Qual 1999;14:160-9.

Publisher's Note Springer Nature remains neutral with regard to jurisdictional claims in published maps and institutional affiliations. 